\title{
Predicting the Location of Glioma Recurrence After a Resection Surgery
}

\author{
E. Stretton ${ }^{1}$, E. Mandonnet ${ }^{2,3,4}$, E. Geremia ${ }^{1}$, B. H. Menze ${ }^{1,5,6}$, \\ H. Delingette ${ }^{1}$ and N. Ayache ${ }^{1}$ \\ ${ }^{1}$ Asclepios Research Project, INRIA, Sophia Antipolis, France, \\ ${ }^{2}$ Hôpital Lariboisière, Neurosurgery Department, Paris, ${ }^{3}$ Université Paris 7 , \\ ${ }^{4}$ IMNC, UMR 8165, Orsay, ${ }^{5}$ CSAIL at MIT, ${ }^{6}$ ETH Zurich
}

\begin{abstract}
We propose a method for estimating the location of glioma recurrence after surgical resection. This method consists of a pipeline including the registration of images at different time points, the estimation of the tumor infiltration map, and the prediction of tumor regrowth using a reaction-diffusion model. A data set acquired on a patient with a low-grade glioma and post surgery MRIs is considered to evaluate the accuracy of the estimated recurrence locations found using our method. We observed good agreement in tumor volume prediction and qualitative matching in regrowth locations. Therefore, the proposed method seems adequate for modeling low-grade glioma recurrence. This tool could help clinicians anticipate tumor regrowth and better characterize the radiologically non-visible infiltrative extent of the tumor. Such information could pave the way for model-based personalization of treatment planning in a near future.
\end{abstract}

\section{Introduction}

Glioma surgical resection has shown to be a critical therapeutic modality and is usually the first type of therapy given to patients. Resections are part of a standard treatment that has demonstrated increased patients' survival time [14]. However, gliomas are a diffuse, infiltrative and resilient form of brain cancer. Most low-grade glioma patients have a tumor recurrence after the first tumor resection. The tumor tends to reoccur most often immediately adjacent to the site of resection despite how extensive the resection [15]. Treatment then includes a second surgery, chemotherapy or radiation therapy, and there is no consensus regarding the best option in this setting. We present a biomathematical tool that would estimate the radiologically non-visible part of the tumor from a longitudinal set of images. Such virtual imaging could potentially guide the clinician in the decision making process (intuitively, surgery should be prefered for a tumor without a large non-visible extent, i.e., the so called "bulky" tumors).

Mathematicians and computer scientists have proposed various methods to tackle portions of this problem $[2,5-7,9,12,13,16,18]$. Clatz et al. [1] and Jbabdi

\footnotetext{
${ }^{1}$ corresponding author: erin.stretton@inria.fr.
} 
et al. [8] proposed DTIs construction methods that estimate the tumor cell diffusion in white matter based on water diffusion in white matter. Konukoglu et al. [9] built upon these models to personalize a tumor growth model to estimate the product of $d_{w, g} * \rho$ (tumor diffusion in white and gray matter multiplied by the tumor proliferation rate). These models allow us to reasonably capture the progression of a tumor for a given patient before a resection or therapy.

The latest work on modeling glioma regrowth following brain tumor surgery was by Swanson et al. [16]. They developed a 3D model of tumor growth accounting for the heterogeneity of brain tissue. In a post mortem study, they investigated the effectiveness of using different types of brain resections. However, their model was limited to personalization using patient T1 Gad and T2 MRIs, without taking into account the anisotropy in white matter fiber tracts visible in diffusion tensor imaging (DTI). In addition, they ran their simulations on virtual controls instead of on patient data.

The pipeline approach that we present in this paper introduces several new features. First, the 3-D simulation results from using our pipeline estimates the most likely location of tumor progression after surgery since tumors do not typically grow at the same rate in all directions. Tumors grow faster in the white matter than in the gray matter of the brain [4]. Therefore, simulating future tumor growth would be very helpful for therapy planning. Second, it estimates the profile of the tumor regrowth, thus informing about the radiologically non-visible extent of the tumor. Third, the simulation results from using our pipeline helps to differentiate hyper-intense voxels between scarring tissue, edema or tumor recurrence. The areas bordering the resection cavity could be flagged as high and

low risk of tumor recurrence areas. Our problem requires solving complex registration problems between pre-op and post-op, combining a tail extrapolation algorithm (to estimate the invisible part of the tumor) with a tumor progression algorithm (to predict future extension). To our knowledge, modeling tumor recurrence after a brain tumor resection using a patient DTI and patient data has not been done before.

This paper is organized into four sections. In Section 2, we describe a method for estimating the location of glioma recurrence. In Section 3, we present the results of our experiments, which show that this method is feasible. In Section 4 , we discuss these results and future work.

\section{Materials and Method}

The proposed method for estimating the location of glioma recurrence after a resection consists of several interconnected steps. The first step entails segmenting the images. The second step consists of a sequence of registrations. The third step is estimating the tumor's infiltration tail on the date of surgery, and the fourth step uses a simulation method to predict the location of tumor regrowth at future time instances. The fifth step allows us to tell if the tumor is a bulky or diffuse tumor. Both the tail extrapolation algorithm and the prediction algo- 
rithm use the same model framework. We tested the proposed approach on data from a clinical study.

Model Framework. Tracqui et al. [17] proposed using reaction-diffusion-based growth models in the form of the Fisher Kolmogorov equation (FK):

$$
\frac{\partial u}{\partial t}=\underbrace{\nabla \cdot(D(\mathbf{x}) \nabla u)}_{\text {Diffusion Term }}+\underbrace{\rho \cdot u \cdot(1-u)}_{\text {Logistic Reaction Term }} ; \underbrace{\eta_{\partial \omega} \cdot(D \nabla u)=0}_{\text {Boundary Condition }}
$$

where $u$ is the tumor cell density, $D$ is the diffusion tensor for tumor cells using the tumor diffusion tensor construction method described below, $\rho$ is the proliferation rate and $\eta_{\partial \omega}$ are the normal directions of the boundaries of the brain surface.

To use this framework, we need a tensor image constructed from a DTI to form $D(\mathbf{x})$, an estimate on parameter values $\left(d_{w}, d_{g}, \rho\right)$, and segmentations of several areas of the brain. $d_{w}$ and $d_{g}$ are scalars that multiply the diffusion tensors.

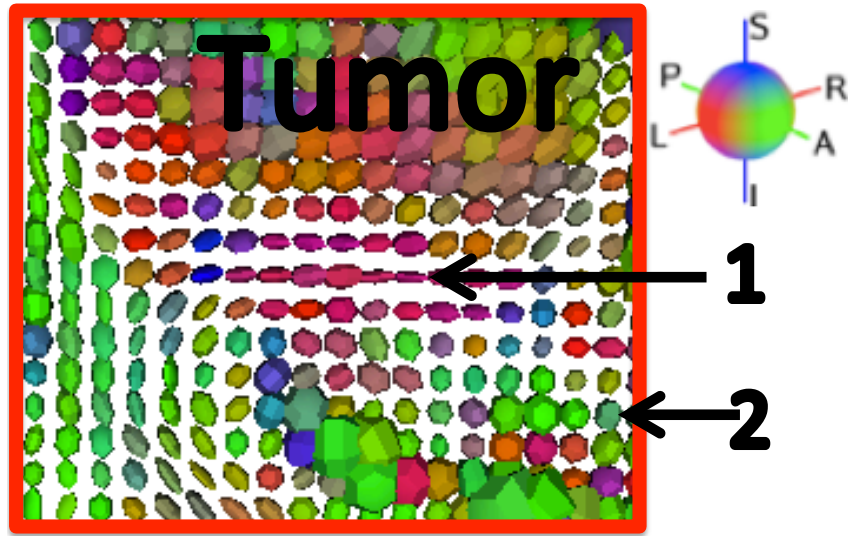

Fig. 1. Day -3 DTI: (1) anisotropic white matter tensors and (2) isotropic gray matter tensors. Region of red box in Figure 2.

There are several tensor construction methods that have been proposed to model anisotropic diffusion $[1,8]$. We used a tensor construction method, proposed by Clatz et al. [1], that uses global scaling on the DTI,

$$
D(\mathbf{x})= \begin{cases}d_{g} I & \text { if } \mathbf{x} \text { is in grey matter } \\ d_{w} D_{\text {water }} & \text { if } \mathbf{x} \text { is in white matter }\end{cases}
$$

where $D(\mathbf{x})$ is the inhomogeneous diffusion term, which takes into account that tumor cells are thought to move faster along anisotropic white matter fiber 
tracts, estimated by $d_{w} D_{\text {water }}$, than in isotropic gray matter $d_{g} I . D_{\text {water }}$ is the water diffusion tensor in the brain measured by the DTI and $I$ is the identity matrix which can be seen as an isotropic diffusion tensor (see Figure 1).

Estimating the parameter values is detailed in the Optimizing $d_{w}$ and $\rho$ Algorithm paragraph.

Assumption. Gliomas appear as hyper-intense voxels in Flair MRIs in which edema appears bright. We assume that where there is edema, there is $20 \%$ or more tumor cell density threshold of visibility. In reality, there might be edema without tumor cells close by and vice versa. Tracqui et al. [17] proposed $40 \%$ maximal tumor cell density to be visible in T2 MRIs, Konukoglu et al. [9] used Tracqui's value, and Swanson et al. [16] used a value of 2\%. Menze et al. [13] suggested the maximal tumor cell density that is visible in Flair MRIs to be $9.5 \%$. We chose the tumor cell density threshold of visibility value as $20 \%$ because it is an intermediate value in literature for T2 MRIs, which includes Flair. Currently, Flair is the imaging modality that shows the most glioma tumor cell density threshold of visibility extents, although distinction with scar tissue or edema is not possible with this sequence.

Data. Our data consists of a current patient with a supra-complete resection and long post-operation (post-op) follow-ups, complements of our collaborating neurosurgeon with informed consent from the patient. It is difficult to acquire this type of longitudinal data, particularly due to limited availability of DTIs. For this reason, the pipeline was only tested on 1 data set. However, this is the first time this data set is being used for research and is not the same data set used by Konukoglu et al. [9] and Clatz et al. [1]. This patient had MRIs acquired on three different dates before surgery and three dates after surgery (see Figure 1). The tumor, resection cavity and tumor regrowth for all of the dates were segmented by the neurosurgeon from Flair MRIs.

The voxel size of our MRIs range from $0.5 \times 0.5 \times 2.0 \mathrm{~mm}^{3}$ to $0.5 \times 0.5 \times$ $5.5 \mathrm{~mm}^{3}$. All images were re-sampled to be $1 \times 1 \times 1 \mathrm{~mm}^{3}$ by resampling the baseline using an in house tool and then registering all images (see Figure 3) to the baseline.

\begin{tabular}{ll}
\hline Interval from & MRIs \\
Surgery Date in Days & Modalities \\
\hline-49 & Flair \\
-3 & DTI \\
-1 & T1 \& Flair \\
+1 & Flair \\
+74 & T1 \& Flair \\
+172 & T1 \& Flair \\
\hline
\end{tabular}

Table 1. Patient MRI acquisition dates. 


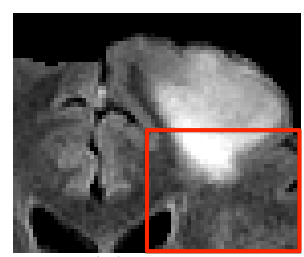

(a) Day -1

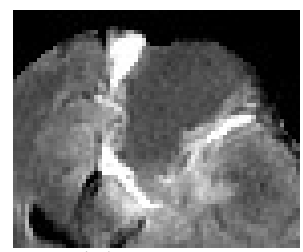

(b) Day +1

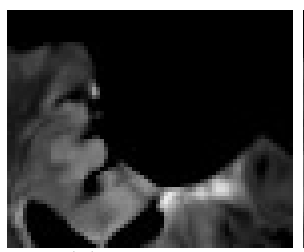

(c) Day +74

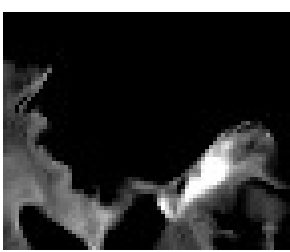

(d) Day +172

Fig. 2. Patient registered Flair MRIs axial views. (a) Hyper-intense region that was considered tumor before resection on Day 0. Red box displays the area shown in Figure 1. (b) Distortion in the resection cavity. Hyper-intense regions were considered scar tissue or hemorrhages caused by surgery for this date. (c) and (d) exhibits hyperintense regions which could be scar tissue, hemorrhages or tumor recurrence. It can be seen with these images that it is not possible to classify these hyper-intense regions and decipher if the tumor recurrence is a bulky or diffuse-type recurring tumor.

Segmentation. The areas of the brain that need to be segmented to clearly define their boundaries are the white and gray matters, the cerebrospinal fluid (CSF) and the tumor at each time point.

The segmentations of white and gray matter are used to mark out the inhomogeneous tissue boundaries used by $d_{w}$ and $d_{g}$. The CSF segmentation is used to define the no flux boundary conditions of the model, i.e., tumor cells cannot enter these masks. To create these segmentations we thresholded white matter and brain parenchyma (white matter + gray matter) probability maps from MNI 152 (Atlas) [3] into binary masks, which recovered all of the necessary sulci structure and separated lobes. This conversion was achieved with the help of the neurosurgeon, who decided the best threshold values for the CSF, gray and white matter probability maps.

The tumor segmentations can be used for three purposes. First, a tumor segmentation is used as the starting boundary where the tumor growth simulation begins. Second, two tumor segmentations at two different time points can be used in a minimization algorithm to find the FK parameters: $d_{w}, d_{g}$, and $\rho$. Third, the following acquisition time point tumor segmentations are used to validate that the simulation results, which were grown from the first time point tumor segmentation, were reasonable.

Registration. The registration sequence employed has several interrelated steps (see Figure 3). The most important part of our registration pipeline is the method we use for nonlinear registration of the images, where there exists no one-to-one correspondence between both images due to the tumor resection or growth. The non-linear deformation between the pre-op images and the post-op images can be assessed with the ventricles swelling and brain tissue shifting position after surgery (even several months after surgery). The idea of the nonlinear registration algorithm employed is to use local confidence weights and to model pathological regions with zero confidence. Lamecker et al. [10] added this algorithm as 


\begin{tabular}{|c|c|c|c|c|c|c|c|}
\hline \multirow{3}{*}{$\begin{array}{c}\text { Atlas } \\
\text { MNI } 152 \text { T1 }\end{array}$} & \multicolumn{5}{|c|}{ Time Pre-Operation } & \multicolumn{2}{|c|}{ Post-Operation } \\
\hline & Day -49 & Day -3 & & Day -1 & Day +1 & Day +74 & Day +172 \\
\hline & Flair & DTI & Flair & & Flair & Flair & Flair \\
\hline $\mathrm{NL}_{1}^{\circ} \mathrm{A}_{1}$ & $\mathrm{R}_{1}$ & $R_{2} L$ & $\stackrel{R_{3} \longrightarrow}{\longrightarrow}$ & BASELNE & & $\neg \uparrow R_{4}$ & $\uparrow R_{5}$ \\
\hline
\end{tabular}

Fig. 3. Registration pipeline where all images are registered to the baseline. $\mathrm{R}$ stands for rigid, A for affine and NL for non-linear registration using a mask. In the non-linear registration we used an inpainting step to register the voxels covered by the mask.

an extension to the efficient and publicly available diffeomorphic demons registration framework. The algorithm requires a mask to cover the areas that cannot be matched between the images (i.e., resection cavity plus tumor volume). This mask volume is excluded from the registration. An inpainting step is used to estimate the registration in the areas covered by the mask.

The registration sequence can be divided into two parts: (1) registration of the atlas-based white matter and brain segmentations (segmentation details are in the Segmentation paragraph), and (2) registration of all patient images and segmentations to the baseline MRI. This registration sequence is depicted in Figure 3 and the results can be seen in Figure 2.

Registering the atlas-based white matter and brain segmentations required two steps. First, we non-linearly registered the Atlas T1 MRI to the patient's baseline MRI (Day -1 T1 re-sampled). Lastly, we applied this displacement field transformation to the white matter and brain binary segmentations.

Registering all of the patient images and segmentations involved three main steps. First, we rigidly registered all pre-op MRIs to the baseline. Next, we removed the skull and did histogram matching on the post-op T1 MRIs before non-linearly registering them to the baseline (the manually segmented mask consisted of the combined pre-op Day -1 tumor and post-op Day +74 resection cavity). Finally, we applied the transformations found registering the post-op T1 MRIs to the post-op Flair MRIs and segmentations.

Tail Extrapolation Algorithm. The third step in the method we are proposing uses the FK equation and the tensor construction method. Konukoglu et al. [9] proposed a static model to overcome the problem of estimating the tumor infiltration tail by extrapolating the tumor invasion margins. The non-linear reaction term in Equation 1 is linearized around $u=0$ and the tail distribution is shown to be asymptotically described by a Hamilton-Jacobi equation of the tumor cell density function. Using a Fast Marching method, an efficient algorithm was proposed that estimates the tumor cell invasion profile outside the visible boundaries in MRIs. For this step, we used the neurosurgeon's Day -1 rigidly registered tumor segmentation, the non-linearly registered white matter and brain segmentations, the rigidly registered DTI, and parameters $d_{w}, d_{g}$ and $\rho$ (see Optimizing $d_{w}$ and $\rho$ Algorithm paragraph for FK parameter choice). As 
the initial condition to this model, we make the assumption of $20 \%$ tumor cell density threshold of visibility (see Assumption paragraph).

Prediction Algorithm. For the fourth step in our method we used this estimated tumor infiltration tail as the initial condition to the FK model (developed by Clatz [1] and Konukoglu et al. [9]) to simulate the location and predicted tumor cell density of recurrence for a given date $[1,9]$. This is done by propagating $u$ by the time defined from MRI acquisition dates. We used two acquisition dates before surgery and one after surgery $(-49,-1$ and +74$)$ to predict where the tumor will grow at the 4 th acquisition date (2nd after surgery). The $d_{w}, d_{g}$ and $\rho$ parameters that were estimated with the first three acquisition times were used.

Optimizing $d_{w}$ and $\rho$ Algorithm. The fifth step in our method was determining which $d_{w}$ and $\rho$ fit each particular patient's data (personalization) since previous algorithms were only able to estimate the velocity constant $\left(v=2 \sqrt{\rho d_{w}}\right)$, but not $d_{w}$ and $\rho$ separately [9,12]. Different values of $d_{w}$ and $\rho$, where $v$ is the same, produce very different overall tumor shapes [9]. For example, if $d_{w} / \rho$ is low, the tumor is said to be bulky (not very infiltrative); where as if $d_{w} / \rho$ is high, the tumor is said to be diffuse. We created a tool to sweep through the physically feasible values, proposed by Harpold et al. [6], of $d_{w}$ and $\rho$ keeping $d_{w} * \rho$ constant. There are two parts to this process: find $v$, and solve for $d_{w}$ and $\rho$.

First, finding $v$ can be done in two different ways. Konukoglu et al. [9] proposed a minimization method for estimating the FK parameters: $d_{w}{ }^{*} \rho$ (differential speed), $d_{g}, T_{0}$ (initial tumor start date). However, for this patient, the tumor does not visibly change volume or shape between Day -49 and Day -1 (possibly due to an overestimation of the tumor extent at Day -49, which was performed soon after a generalized seizure). We used the second way of finding $v$, which was to assume that the diameter velocity of the tumor was $4 \mathrm{~mm} /$ year, which was proposed by Mandonnet et al. in [11] for low grade glioma tumor growth.

Then, to solve for $d_{w}$ and $\rho$, we swept through the possible parameter values of $d_{w}$ (4 to $10 \mathrm{~mm}^{2} /$ year) and $\rho$ (0.4 to 1.01 /year), keeping $v$ constant at $4 \mathrm{~mm} /$ year, iterating through steps 2 and 3 of our method. We started the Tail Extrapolation Algorithm from Flair segmentation Day -1 with resection cavity removed from the image to compare with Flair segmentation Day +74 . We found the value of $d_{w}=6 \mathrm{~mm}^{2} /$ year and $\rho=0.6671 /$ year to be the most appropriate by qualitative analysis. These values of $d_{w}$ and $\rho$ were used to predict Flair segmentation Day +172 and the results are discussed in the Results section (also see Figure 4).

The parameters that determine the shape of the tumor, which are perceptible only locally in white matter, are the tensor construction method and the ratio $d_{w} / d_{g}$, where $d_{w} / d_{g}=1$ is isotropic growth and $d_{w} / d_{g}=100$ is highly anisotropic growth. There are two ways of finding $d_{g}$ : using a minimization algorithm, such as the one proposed by Konukoglu et al. [9], or sweeping through the possible values of $d_{g}$, once you have found $d_{w}$ and $\rho$, by iterating through steps 2 and 


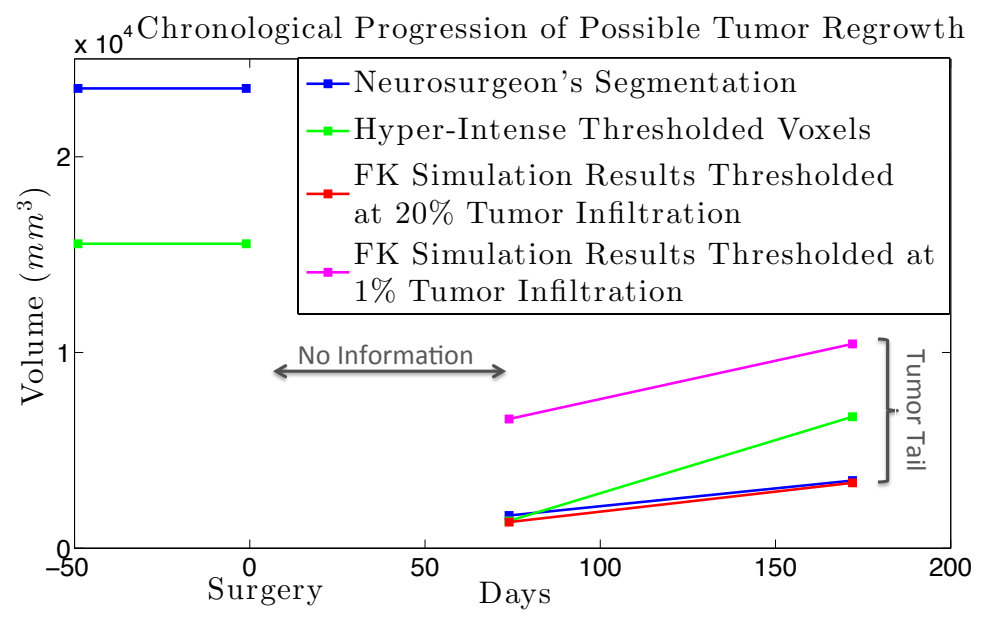

Fig. 4. For Day +74 , there is an agreement in volume for 3 of the segmentations. For Day +172 , both the neurosurgeon's estimation of tumor growth and the FK prediction match in volume.

3 of our method. Since we were not able to use a minimization algorithm on this patient's data, due to a likely seizure-induced overestimation of real tumor size at first MRI, we swept through the the values of $d_{g}\left(1\right.$ to $6 \mathrm{~mm}^{2} /$ year $)$. We found $d_{g}=1 \mathrm{~mm}^{2} /$ year to be the most appropriate value by qualitative analysis.

\section{Results}

In this paper, we have proposed a method to predict where tumor regrowth will occur for glioma resection patients.

Quantitatively, Figure 4 shows the chronological progression of the patient's possible tumor regrowth. Due to the large amount of brain shift plus tumor evolution in the post-op MRIs, the non-linear registration compresses and stretches the tissues surrounding the resection cavity. For this reason we believe matching volumes and not surfaces is reasonable. Using overlap measures would imply to perform voxel to voxel comparison between pre-op and post-op images. This is a very challenging registration problem due to the large deformations caused by the tumor removal. As the registration errors are still large in those areas, we chose to compare the tumor segmentation and prediction by using global measures (volumes) rather than local measures like overlap. We use two criteria for evaluating our method's accuracy: the neurosurgeon's segmentations and hyper-intense signal segmentations. The hyper-intense signal segmentation shows all the voxels that could possibly be tumor due to their intensity in the image. Results show that for Day +74 , there is a good volume agreement for 3 of the segmentations. For Day +172 , both the neurosurgeon's estimation of tumor 


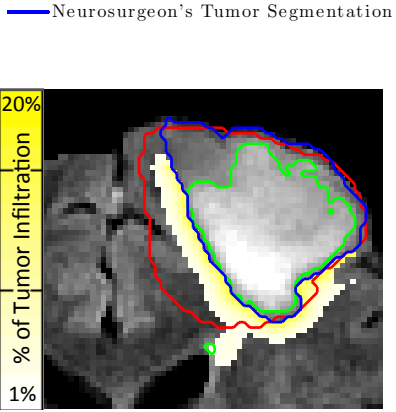

(a) Day -1

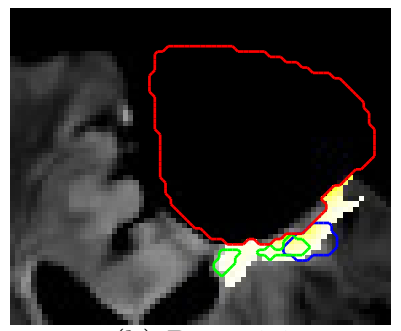

(b) Day +74

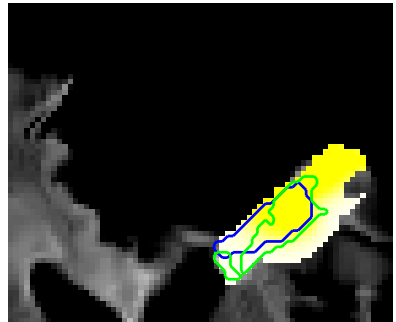

(c) Day +172

Fig. 5. Compare this figure with Figure 1 and 2. (a) Shows the estimated tumor infiltration tail in yellow. This tail cannot be distinguished with current MRI technology. In (b) and (c), the simulated tumor regrowth predictions are shown in yellow. Observe that the hyper-intense regions do not exactly cover the same regions as were flagged by the neurosurgeon (blue). However, both areas are covered by the prediction of $1 \%$ tumor infiltration.

growth and the FK simulated prediction (thresholded at $20 \%$ tumor infiltration) match in volume. This demonstrates that the model includes the visible part of the tumor in its prediction, but also flags areas which are not visible with current MRI technology (shown in magenta). Additionally, the agreement in volume between the neurosurgeon's segmentation and the model's results signifies that the tumor location outlined by the neurosurgeon is not a simple function of signal intensity.

Qualitatively, we show in Figure 5 that our model provides a reasonable estimate of the tumor infiltration tail after resection. Figures 2 and 1 display the same axial slice and should be used to aid interpretation of this figure. In Figure 5(a) we show the estimated tumor infiltration tail that cannot be seen in MRI images (step 2 of our method). In Figure 5(b) and (c), the predicted tumor regrowth is displayed in yellow (step 3 of our model). We can see from Figure 5(a) that the tumor tail (1\% tumor infiltration) was not removed with the brain resection. This tail was the seed of regrowth, which is evident in the Day +74 and Day +172 MRIs. If we compare Figure 5(b) and (c) with Figure 1, we can see that the patient's white matter tensors, which are bordering the resection cavity, are anisotropic. These tensor's shape were a large contributor to dictating the speed and direction in which the tumor was simulated to grow. The green lines outline the hyper-intense voxels in the Flair MRIs. These regions could be scarring and/or edema caused by surgery and/or tumor recurrence cell density above or equal to $20 \%$ of maximal cell density. The blue line was classified by the neurosurgeon as possible tumor. Observe that the hyper-intense regions do not exactly cover the same regions as were flagged by the neurosurgeon. However, both areas are covered by the FK simulated prediction at the tumor cell density threshold of visibility value of $1 \%$. 
Depending on the size and resolution of the image, the automated process of registration, estimating the tumor infiltration map and simulating future tumor regrowth sites for one future time instance can take about 20 hours on a single CPU running at $2.2 \mathrm{GHz}$. The main time-consuming step is the non-linear registration with inpainting.

\section{Discussion and Conclusion}

We presented an approach to predict tumor regrowth after a brain tumor resection. We used a novel pipeline combining image registration with a static model for estimating the tumor infiltration tail and a dynamic simulation model for predicting future tumor regrowth. Our results show that predicting is possible for future tumor regrowth using a reaction-diffusion-type model that employs a patient DTI.

The non-linear registration step that we employed was key in making our method possible. Other non-linear registration methods, such as demons (without extensions) or pyramidal block-matching algorithms that use masks, were not able to deal with the resection cavity to tumor registration. The non-linear registration step that we used was designed to work with an atlas to patient registration in the presence of pathologies in the patient image. Although it worked quite well for the tumor resection application, we could improve the registration results if we extended this algorithm to use more specific prior information for resection images.

In the future, we intend to study more glioma resection patients having regrowth after surgery using this method. We will study all of the parameter interactions of our method, as well as explore using other tensor construction techniques for the tail extrapolation algorithm and prediction algorithm parts of our method, e.g. Jbabdi et al. [8]. Since glioma growth modeling is patientspecific, we intend to improve our method and validate it using a large patient data set. This data set will help us analyze the best way to improve the registration, minimization of parameters and investigate if the tumor growth rate stays constant after a tumor resection, as seen previously among numerous patients. With a large number of patients studied, we will develop a method to predict more precisely these parameters separately, prior to a glioma resection. This will enable the model to more precisely predict where the tumor could reoccur after surgery.

\section{Acknowledgments}

This work was partially supported by the Care4me ITEA2 project and ERC MedYMA. A big thank you to Maxime Sermesant, Jatin Relan and Gregoire Malandain for their support. 


\section{References}

1. O. Clatz, M. Sermesant, P.Y. Bondiau, H. Delingette, S.K. Warfield, G. Malandain, and N. Ayache. Realistic simulation of the 3-d growth of brain tumors in $\mathrm{mr}$ images coupling diffusion with biomechanical deformation. Medical Imaging, IEEE Transactions on, 24(10):1334-1346, 2005.

2. D. Cobzas, P. Mosayebi, A. Murtha, and M. Jagersand. Tumor invasion margin on the riemannian space of brain fibers. Medical Image Computing and ComputerAssisted Intervention-MICCAI 2009, pages 531-539, 2009.

3. VS Fonov, AC Evans, RC McKinstry, CR Almli, and DL Collins. Unbiased nonlinear average age-appropriate brain templates from birth to adulthood. Neuroimage, 47:S102-S102, 2009.

4. A. Giese and M. Westphal. Treatment of malignant glioma: a problem beyond the margins of resection. Journal of cancer research and clinical oncology, 127(4):217$225,2001$.

5. A. Gooya, G. Biros, and C. Davatzikos. Deformable registration of glioma images using em algorithm and diffusion reaction modeling. IEEE Trans Med Imaging, 30(2):375-390, Feb 2011.

6. H.L.P. Harpold, E.C. Alvord Jr, and K.R. Swanson. The evolution of mathematical modeling of glioma proliferation and invasion. Journal of Neuropathology $\mathcal{G}^{3}$ Experimental Neurology, 66(1):1, 2007.

7. C. Hogea, C. Davatzikos, and G. Biros. An image-driven parameter estimation problem for a reaction-diffusion glioma growth model with mass effects. J. of Math. Bio., 56(6):793-825, 2008.

8. S. Jbabdi, E. Mandonnet, H. Duffau, L. Capelle, K.R. Swanson, M. Pélégrini-Issac, R. Guillevin, and H. Benali. Simulation of anisotropic growth of low-grade gliomas using diffusion tensor imaging. MRM, 54(3):616-624, 2005.

9. E. Konukoglu, O. Clatz, B.H. Menze, B. Stieltjes, M.A. Weber, E. Mandonnet, H. Delingette, and N. Ayache. Image guided personalization of reaction-diffusion type tumor growth models using modified anisotropic eikonal equations. Medical Imaging, IEEE Transactions on, 29(1):77-95, 2010.

10. H. Lamecker, X. Pennec, et al. Atlas to image-with-tumor registration based on demons and deformation inpainting. In Proc. MICCAI Workshop on Computational Imaging Biomarkers for Tumors-From Qualitative to Quantitative, CIBT. Citeseer, 2010.

11. E. Mandonnet, J.Y. Delattre, M.L. Tanguy, K.R. Swanson, A.F. Carpentier, H. Duffau, P. Cornu, R. Van Effenterre, E.C. Alvord Jr, and L. Capelle. Continuous growth of mean tumor diameter in a subset of grade ii gliomas. Annals of neurology, 53(4):524-528, 2003.

12. B. H. Menze, E. Stretton, E. Konukoglu, and N. Ayache. Image-based modeling of tumor growth in patients with glioma. In Optimal control in image processing. Springer, Heidelberg/Germany, 2011.

13. B. H. Menze, K. Van Leemput, A. Honkela, E. Konukoglu, M.A. Weber, N. Ayache, and P. Golland. A generative approach for image-based modeling of tumor growth. In Information Processing in Medical Imaging, pages 735-747. Springer, 2011.

14. N. Sanai and M.S. Berger. Glioma extent of resection and its impact on patient outcome. Neurosurgery, 62(4):753, 2008.

15. R. Sawaya. Extent of resection in malignant gliomas: a critical summary. Journal of Neuro-Oncology, 42(3):303-305, 1999. 
16. KR Swanson, RC Rostomily, and EC Alvord. A mathematical modelling tool for predicting survival of individual patients following resection of glioblastoma: a proof of principle. British journal of cancer, 98(1):113-119, 2007.

17. P. Tracqui, GC Cruywagen, DE Woodward, GT Bartoo, JD Murray, and EC Alvord Jr. A mathematical model of glioma growth: the effect of chemotherapy on spatio-temporal growth. Cell Proliferation, 28(1):17-31, 1995.

18. E. Zacharaki, C. Hogea, D. Shen, G. Biros, and C. Davatzikos. Non-diffeomorphic registration of brain tumor images by simulating tissue loss and tumor growth. Neuroimage, 46:762-774, 2009. 